Please do not remove this page

RMIT

UNIVERSITY

\title{
Reverse engineering of a fixed wing unmanned aircraft 6-DoF model based on laser scanner measurements
}

Burston, Martin; Sabatini, Roberto; Gardi, Alessandro Giacomo Maria; Clothier, Reece

https://researchrepository.rmit.edu.au/esploro/outputs/9921862409201341/filesAndLinks?institution=61RMIT_INST\&index=null

Burston, M., Sabatini, R., Gardi, A. G. M., \& Clothier, R. (2014). Reverse engineering of a fixed wing unmanned aircraft 6-DoF model based on laser scanner measurements. Proceedings of 2014 IEEE International Workshop on Metrology for Aerospace, MetroAeroSpace 2014, 144-149.

https://doi.org/10.1109/MetroAeroSpace.2014.6865910

Document Version: Accepted Manuscript

Published Version: https://doi.org/10.1109/MetroAeroSpace.2014.6865910

Repository homepage: https://researchrepository.rmit.edu.au

(C) 2014 IEEE

Downloaded On 2023/04/26 21:44:13 +1000

Please do not remove this page 
Thank you for downloading this document from the RMIT Research Repository.

The RMIT Research Repository is an open access database showcasing the research outputs of RMIT University researchers.

RMIT Research Repository: http://researchbank.rmit.edu.au/

\section{Citation:}

Burston, M, Sabatini, R, Gardi, A and Clothier, R 2014, 'Reverse engineering of a fixed wing unmanned aircraft 6-DoF model based on laser scanner measurements', in Graham Wild, Roberto Sabatini (ed.) Proceedings of 2014 IEEE International Workshop on Metrology for Aerospace, MetroAeroSpace 2014, United States, 29-30 May 2014, pp. 144-149.

See this record in the RMIT Research Repository at:

https://researchbank.rmit.edu.au/view/rmit:28751

Version: Accepted Manuscript

Copyright Statement: (C) 2014 IEEE

Link to Published Version:

http://dx.doi.org/10.1109/MetroAeroSpace.2014.6865910 


\title{
Reverse Engineering of a Fixed Wing Unmanned Aircraft 6-DoF Model Based on Laser Scanner Measurements
}

\author{
Martin Burston, Roberto Sabatini, Alessandro Gardi and Reece Clothier \\ RMIT University - SAMME, Melbourne, Australia \\ roberto.sabatini@rmit.edu.au
}

\begin{abstract}
This paper describes a method for deriving sixdegree-of-freedom (6-DoF) aircraft dynamics parameters adopting reverse engineering techniques from three dimensional (3D) laser scanner measurements. In particular, the mass and aerodynamic properties of the JAVELIN Unmanned Aircraft (UA) are determined using accurate measurements from the 3D scanner and successive CAD processing of the geometric data. In order to qualitatively assess the calculated 6-DoF, the trajectory for the spiral mode excited by the engine torque of this $U A$ is simulated and compared to that of a published 6-DoF of the popular AEROSONDE UA which has very similar geometry. Additionally, to further confirm the validity of the approach, the reverse engineering procedure is applied to a published CAD model of the AEROSONDE UA and the associated 6-DoF parameters are calculated. Using these parameters, a spiral descent trajectory is generated using both the published and calculated parameters. The trajectories match closely, providing a good qualitative verification of the reverse engineering method. In future research, the accurate knowledge of the 6-DoF dynamics will enable the development of an Aircraft Dynamics Model (ADM) virtual sensor to augment the UA navigation system in case of primary navigation sensor outages. Additionally, further refinement of the calculated 6-DoF will involve wind tunnel and flight testing activities.
\end{abstract}

Keywords-6-DOF; flight dynamics; laser scanner; reverse engineering; unmanned aircraft

\section{INTRODUCTION}

The adoption of Unmanned Aircraft (UA) for commercial applications is growing at an unprecedented rate, especially in Australia where the number of UA operator certificates doubles every year [1]. This has led Australian universities to engage in research for many of these applications, utilizing both rotary and fixed wing aircraft. This paper presents an approach whereby a sixdegree-of-freedom (6-DoF) dynamics model is developed without reference to original design drawings and manufacturer detained information. Reverse-engineering techniques are used to retrieve detailed geometric information about the UA airframe, which can be converted into information about the mass properties of the UA [2]. The retrieved 6-DoF model will be successively implemented onboard the UA as a virtual sensor in a similar manner to that proposed in [3] to compensate for the shortcomings of low cost IMU's in high-dynamics attitude determination tasks. Henceforth, the term 'virtual sensor' is used to describe a system akin to that presented in [3] and
[4], where an aircraft dynamics model, in addition to Inertial Navigation System (INS), Global Navigation Satellite System (GNSS) and vision-based sensors (when employed), provides additional inputs for an Extended Kalman Filter (EKF) fusion and estimation architecture.

\section{REVERSE ENGINEERING METHODOLOGY}

The reverse engineering methodology presented in this paper involves development of a virtual model, or mockup, of the UA. This is performed using 3D scanning measurements and then reconstructing the model in a computer-aided design (CAD) package. The next step, developing the 6-DoF model, involves determining the mass properties of the UA from the virtual mockup in CAD. Determination of aerodynamic coefficients and derivatives can be performed in parallel with this step using point measures of the airframe. The final step involves validating the dynamics model, first qualitatively by comparison of its simulated trajectory to that of a verified 6-DoF published in literature. Further quantitative verification and refinements are performed using flight tests. For this research, we used the most recent addition to RMIT University UA research fleet, the JAVELIN, a fixed wing UA with an estimated MTOW of less than $20 \mathrm{~kg}$. Table I shows the comparison between the JAVELIN and a similar UA, the AEROSONDE Mk1. Additionally, Table II lists the characteristics of the Handyscan EXAscan 3D laser scanner used to obtain 3D surface geometry data of the UA.

TABLE I. CHARACTERISTICS OF THE AEROSONDE MK1 AND THE JAVELIN UA [5] [6]

\begin{tabular}{|c|c|c|}
\hline Parameter & AEROSONDE UA & JAVELIN UA \\
\hline Length [mm] & 1700 & 2000 \\
\hline Height [mm] & 600 & 650 \\
\hline Wingspan [mm] & 2900 & 2800 \\
\hline Aspect Ratio & 15 & 11 \\
\hline Empty Weight [kg] & 8.5 & 8.7 \\
\hline MTOW [kg] & 13.5 & $15+$ \\
\hline Fuel Capacity [L] & 5 & 2 \\
\hline Endurance [hours] & $30+$ & $\sim 3$ \\
\hline Engine & $20 \mathrm{cc}$ & $20 \mathrm{cc}$ \\
\hline Max Power $[\mathrm{kW}]$ & 1.28 & 1.28 \\
\hline
\end{tabular}

The 3D scanner was connected to a laptop computer for real time processing and display of the point cloud data. In 
order for the $3 \mathrm{D}$ scanner to determine its position relative to the surfaces it scans, the scanned object required a covering of a random arrangement of reflective stickers (dots). The randomness of the placement of the dots was essential as the scanner relied on the unique patterns of groups of 4-5 dots in order to locate itself as seen in Fig. 1.

TABLE II. CHARACTERISTICS OF THE HANDYSCAN EXASCAN 3D SCANNER [7]

\begin{tabular}{|c|c|}
\hline Parameter & Value \\
\hline Weight & $1.25 \mathrm{~kg}$ \\
\hline Dimensions & $172 \times 260 \times 216 \mathrm{~mm}$ \\
\hline Measurement Rate & 25000 measures $/ \mathrm{s}$ \\
\hline Laser Class & II (eye-safe) \\
\hline Resolution & $0.050 \mathrm{~mm}$ \\
\hline Accuracy & Up to $0.040 \mathrm{~mm}$ \\
\hline
\end{tabular}

The JAVELIN was scanned in sections, with each section being scanned and saved independently of the others. The partially modular nature of the UA was also exploited to allow scanning to be completed in stages, with the wings being physically detached and scanned separately. The point data obtained from the scanner were then processed into geometric surfaces using the reverse engineering software Geomagic as seen in Fig. 2.

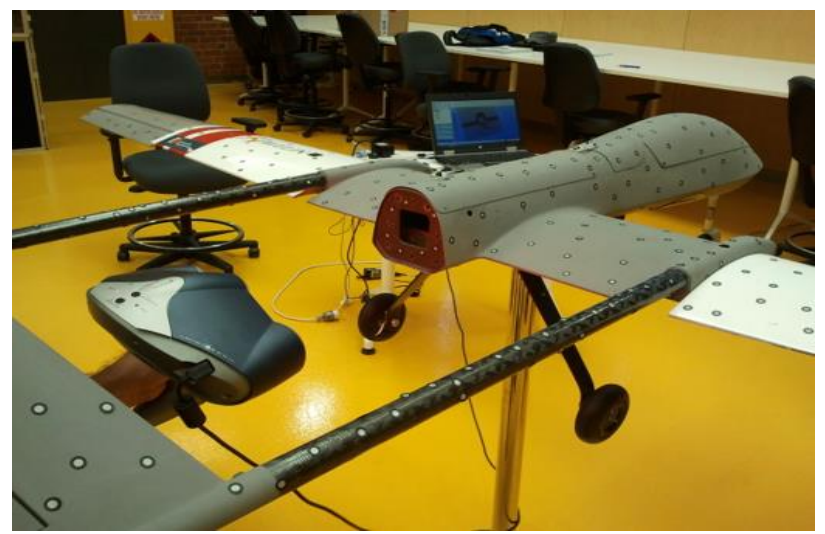

Fig. 1. 3D scanning of the JAVELIN.

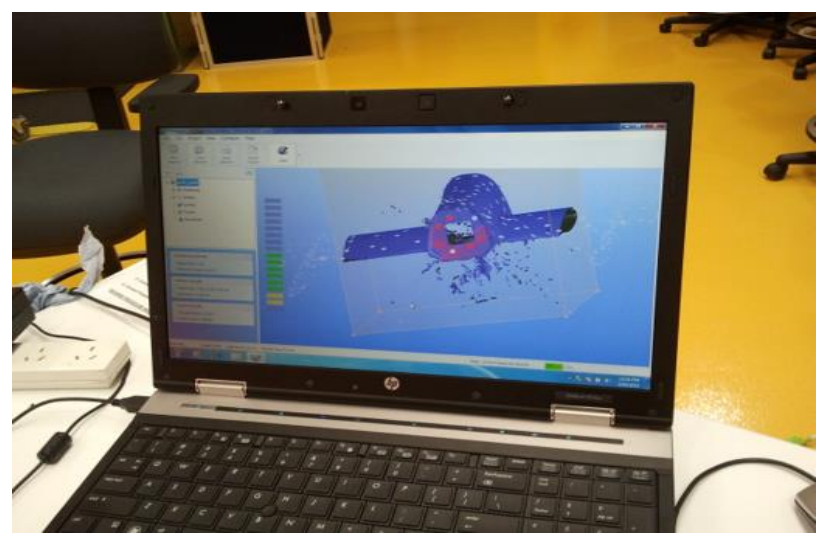

Fig. 2. Resulting 3D rendering.
With Geomagic software, the geometric surfaces were then exported to a CAD program - in this case CATIA V5. The landing gear was included in the initial scans and was modelled manually using conventional measurements. The imported parts had to be manually aligned with the global axis system. The 3D scan files provided accurate external geometry of the airframe - in most cases with less than $1 \%$ deviation from the true distances in the geometry, but no data was obtainable for internal structures due to the inaccessibility of those areas to the scanner, so manual editing of the parts was required to obtain correct skin thicknesses - a crucial component of this project if aircraft moments of inertia were to be determined from the digital model. The skin thicknesses were measured using conventional point measurement techniques. Editing the scanned parts proved difficult due to large file size and unrecognized geometry types. A more traditional approach to reverse engineering was used, where three-view engineering drawings of the scanned files provided guidelines for reconstruction of the parts using geometric functions in CATIA as illustrated in Fig. 3 and 4.

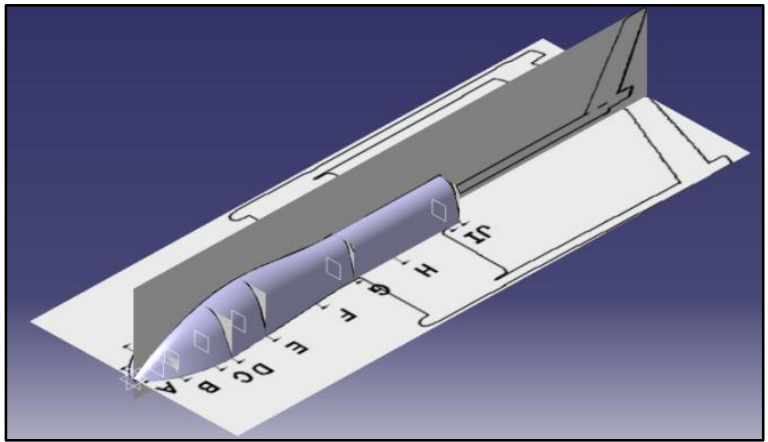

Fig. 3. Reconstruction of the fuselage in CATIA

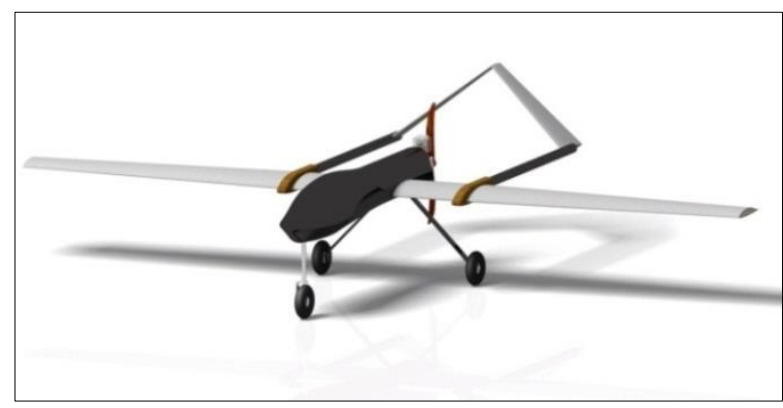

Fig. 4. Finalized 3D CAD of the JAVELIN

The center of gravity $(\mathrm{CoG})$ location and mass moments of inertia of the JAVELIN about its axes were determined using the assembly of all of the digitized components in CATIA. By splitting parts into sub-parts, each of which represents the section of the parent part and is a single homogenous material, an approximation of the distribution of mass throughout the airframe was achieved. Furthermore, the CATIA model facilitated exploration of a range of possible mass distributions using mass models of key subsystems such as the engine, fuel tank, avionics stack and payload. 


\section{DEVELOPMENT OF 6-DOF DYNAMICS}

Once the mass properties of the JAVELIN had been obtained, a 6-DoF theoretical model could be developed which would model the response of the aircraft to disturbances and control inputs. The 6-DoF dynamic model for the JAVELIN is derived using equations of motion for a fixed-wing aircraft as in [8]. In order to implement these equations, the aerodynamic derivatives and coefficients are required first. These were determined using a mixture of techniques - the majority of stability and control derivatives were calculated using empirical equations originally developed for DATCOM as discussed in [8]. Sensitive coefficients such as zero-alpha lift and lift-curve slope were calculated using a 3D vortex lattice method in order to achieve higher accuracy for the given UA geometry, which may vary significantly from what is accounted for by the empirical formulae. The JAVELIN UA model in XFLR5 following the $3 \mathrm{D}$ vortex lattice analysis is illustrated in Fig. 5 .

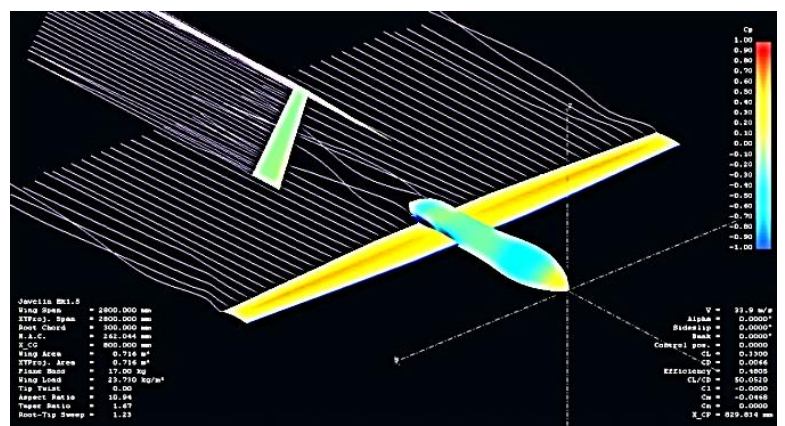

Fig. 5. The JAVELIN UA model in XFLR5 following the 3D vortex lattice analysis.

A preliminary analysis of the calculated derivatives and coefficients was performed by comparing the calculated parameters of the JAVELIN with the validated parameters for a geometric and inertially similar UA - the AEROSONDE Mk1, the parameters for which are provided with the AeroSim Blockset for Simulink [5]. A comparison of several stability derivatives, separated into groups and ordered by importance by [8] are given in Table III. In addition to the aerodynamic coefficients, the moments of inertia are required to complete the 6-DoF dynamics model. These are determined from the detailed CAD model developed earlier, and are compared to the values of the AEROSONDE. Significant similarities can be seen from Table III. Although not a true validation, this comparison does provide some indication that the method provides reasonable values. Experimentation is required to verify and improve the accuracy of the aerodynamic coefficients. For this simulation, it is assumed that the propulsion model for the JAVELIN is the same as that of the AEROSONDE. This decision was made due to a lack of data for other propulsion systems, and future simulations will focus on developing a native propulsion model for the JAVELIN.

\section{6-DoF MODEL VALIDATION}

In order to validate the JAVELIN 6-DoF model without the use of flight tests, a comparative approach is considered, whereby an open-loop simulation is performed for both the JAVELIN and the AEROSONDE UA. The simulation begins with each aircraft at $250 \mathrm{~m}$ ASL $(230 \mathrm{~m}$ AGL at the location of the simulation), with the throttle set to $40 \%$. Although the aircraft are trimmed for steady-level flight, without control inputs the unbalanced torque from the engine excites the spiral mode and the aircraft begin to descent in a helix trajectory. The simulations are stopped when the aircraft reach the ground. As can be deduced from Fig. 6, the JAVELIN UA reaches the ground more quickly than the AEROSONDE. As they descend, the bank angle of the aircraft converges to a stable value, which is around $-60^{\circ}$ for both UA as illustrated in Fig. 7. Due to a greater bank angle and a shorter time to reach the ground, it can be seen in Fig. 8 that the JAVELIN completes fewer loops before it reaches the groundFig. . Further qualitative verification of this method is undertaken by comparing the trajectories plotted using the aerodynamic derivatives of the AEROSONDE as published by Aerosim [5] and the same derivatives calculated from a CAD model of the AEROSONDE. The estimated $C_{n_{\beta}}$ appears significantly larger than the published value, and this can be effectively noticed in the spiral mode, which is sensitive to this parameter. For these comparisons, aircraft mass properties and the propulsion model were the same. 
TABle III. Stability Derivatives AND Moments of INERTIA FOR THE AEROSONDE AND THE JAVELIN [5] [8]

\begin{tabular}{|c|c|c|c|c|}
\hline Parameter & Units & AEROSONDE & JAVELIN & Determination method \\
\hline$C_{L_{\alpha}}$ & $/ \mathrm{rad}$ & 5.61 & 4.97 & 3D vortex lattice using [9] \\
\hline$C_{m_{\alpha}}$ & $/ \mathrm{rad}$ & -2.74 & -1.57 & 3D vortex lattice using [9] \\
\hline$C_{l_{\beta}}$ & $/ \mathrm{rad}$ & -0.13 & -0.073 & Empirical [8] \\
\hline$C_{n_{\beta}}$ & $/ \mathrm{rad}$ & 0.073 & 0.072 & Empirical [8] \\
\hline$C_{m_{\dot{\alpha}}}$ & $/ \mathrm{rad}$ & -10.38 & -2.05 & Empirical [8] \\
\hline$C_{m_{q}}$ & $/ \mathrm{rad}$ & -38.21 & -7.24 & Empirical [8] \\
\hline$C_{l_{p}}$ & $/ \mathrm{rad}$ & -0.51 & -0.46 & Empirical [8] \\
\hline$C_{n_{r}}$ & $/ \mathrm{rad}$ & -0.095 & -0.075 & 3D vortex lattice using [9] \\
\hline$C_{D_{\min }}$ & $/ \mathrm{rad}$ & 0.043 & 0.03 & 3D vortex lattice using [9] \\
\hline$C_{L_{0}}$ & $/ \mathrm{rad}$ & 0.23 & 0.33 & 3D vortex lattice using [9] \\
\hline$C_{m_{0}}$ & $/ \mathrm{rad}$ & 0.135 & 0.135 & Empirical [8] \\
\hline$C_{L_{\dot{\alpha}}}$ & $/ \mathrm{rad}$ & 1.97 & 0.62 & Empirical [8] \\
\hline$C_{L_{q}}$ & $/ \mathrm{rad}$ & 7.95 & 6.70 & CAD model \\
\hline$I_{x x}$ & $\mathrm{kgm}^{2}$ & 0.824 & 1.647 & CAD model \\
\hline$I_{y y}$ & $\mathrm{kgm}^{2}$ & 1.135 & 1.531 & CAD model \\
\hline$I_{z z}$ & $\mathrm{kgm}^{2}$ & 1.759 & 3.094 & 0.066 \\
\hline$I_{x z}$ & $\mathrm{kgm}^{2}$ & 0.120 & \multicolumn{2}{c|}{} \\
\hline
\end{tabular}

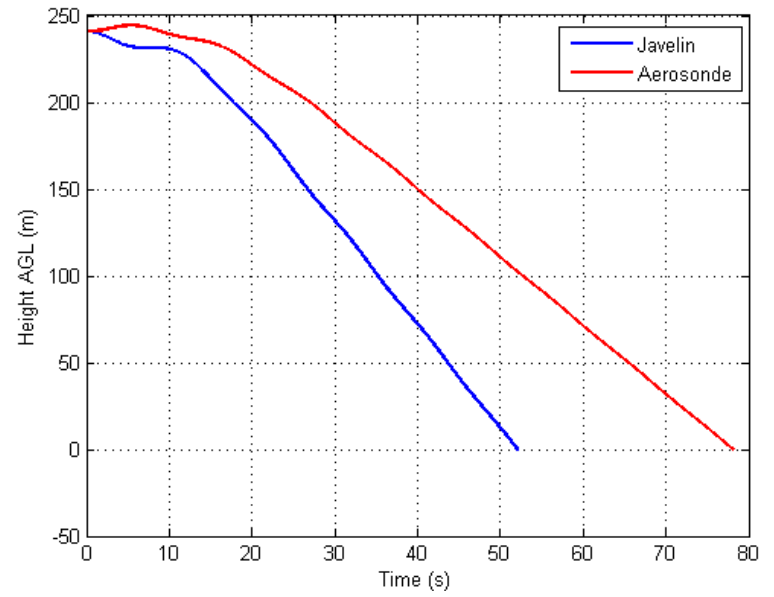

Fig. 6. Comparison of JAVELIN and AEROSONDE UA altitude profiles in the spiral trajectories.

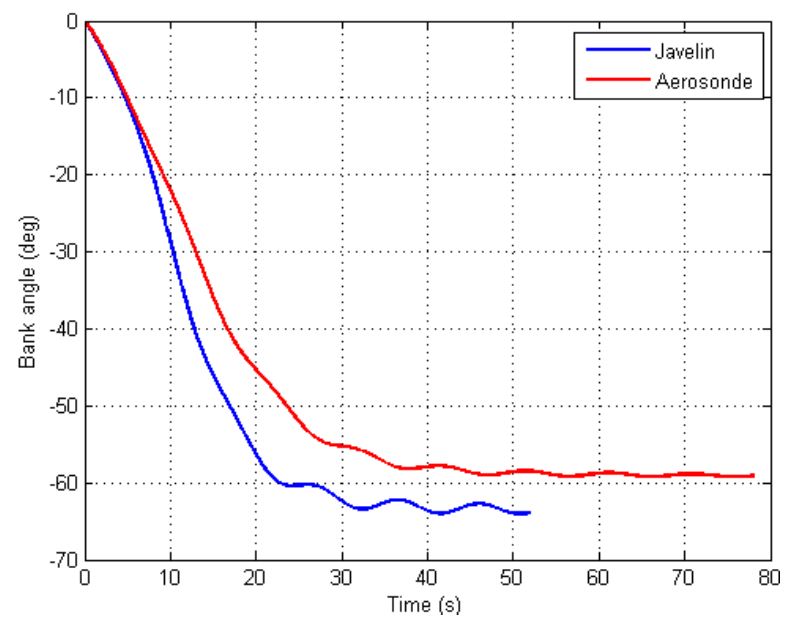

Fig. 7. Comparison of JAVELIN and AEROSONDE UA bank angle profiles in the spiral trajectories.

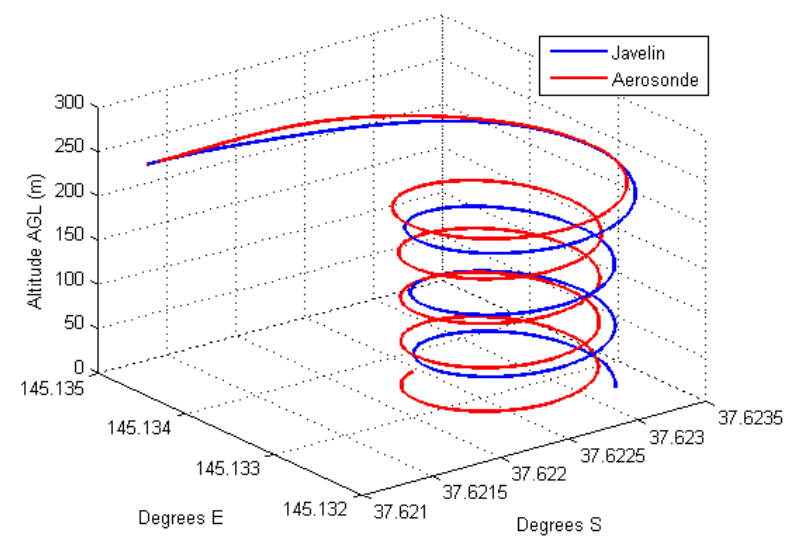

Fig. 8. Spiral trajectories of JAVELIN and AEROSONDE UA.

The performance of the calculated 6-DoF for the AEROSONDE UA is similar to that of the published 6-DoF when lateral, longitudinal and airspeed closed-loop PI \& PID control methods are implemented. The comparison of published and calculated AEROSONDE 6-DoF is illustrated in Fig. 9. The altitude and the bank angle profiles are seen to match closely as illustrated in Fig. 10 and 11.

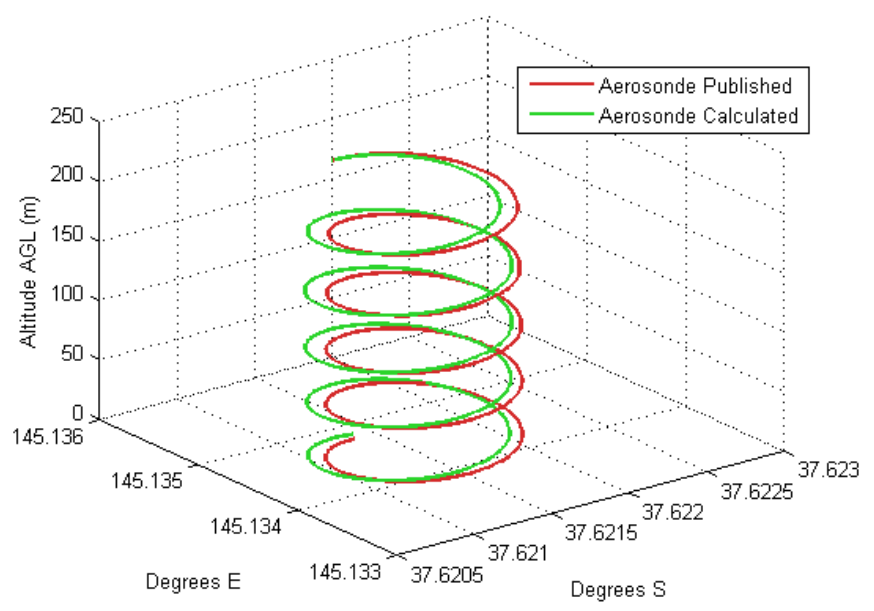

Fig. 9. Comparison of published and calculated AEROSONDE 6-DoF in controlled spiral descent trajectories. 


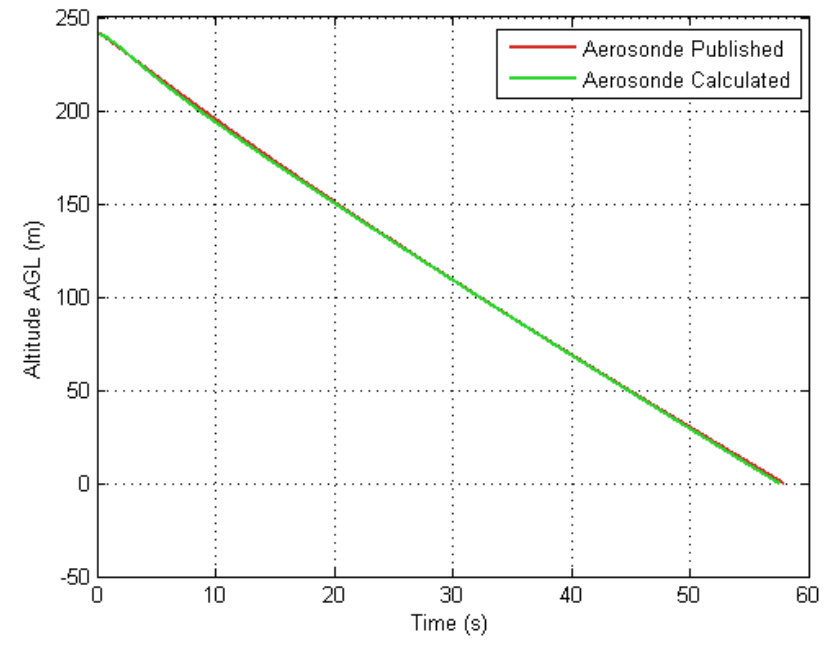

Fig. 10. Altitude profiles for the AEROSONDE 6-DoF spiral trajectories.

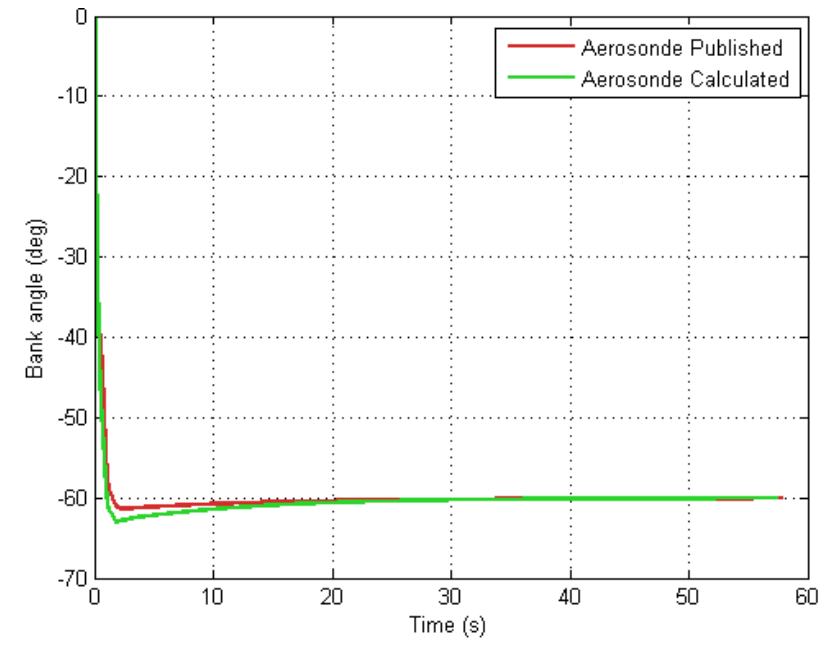

Fig. 11. Bank angle (right) profiles for the AEROSONDE 6-DoF spiral trajectories.

A comparison of the key published and calculated aerodynamic coefficients for the AEROSONDE UA are given in Table IV.

TABle IV. Comparison of Published and CAlCulated Stability Derivatives For AEROSONDE UA [5] [8]

\begin{tabular}{|c|c|c|c|c|}
\hline Parameter & Units & AEROSONDE published & AEROSONDE calculated & Determination method \\
\hline$C_{L_{\alpha}}$ & $/ \mathrm{rad}$ & 5.61 & 5.21 & 3D vortex lattice using [9] \\
\hline$C_{m_{\alpha}}$ & $/ \mathrm{rad}$ & -2.74 & -3.56 & 3D vortex lattice using [9] \\
\hline$C_{l_{\beta}}$ & $/ \mathrm{rad}$ & -0.13 & -0.16 & Empirical [8] \\
\hline$C_{n_{\beta}}$ & $/ \mathrm{rad}$ & 0.073 & 0.15 & Empirical [8] \\
\hline$C_{m_{\dot{\alpha}}}$ & $/ \mathrm{rad}$ & -10.38 & -4.18 & Empirical [8] \\
\hline$C_{m_{q}}$ & $/ \mathrm{rad}$ & -38.21 & -19.91 & Empirical [8] \\
\hline$C_{l_{p}}$ & $/ \mathrm{rad}$ & -0.51 & -0.47 & Empirical [8] \\
\hline$C_{n_{r}}$ & $/ \mathrm{rad}$ & -0.095 & -0.14 & 3D vortex lattice using [9] \\
\hline$C_{D_{\min }}$ & $/ \mathrm{rad}$ & 0.043 & 0.03 & 3D vortex lattice using [9] \\
\hline$C_{L_{0}}$ & $/ \mathrm{rad}$ & 0.23 & 0.23 & 3D vortex lattice using [9] \\
\hline$C_{m_{0}}$ & $/ \mathrm{rad}$ & 0.135 & 0.135 & Empirical [8] \\
\hline$C_{L_{\dot{\alpha}}}$ & $/ \mathrm{rad}$ & 1.97 & 0.84 & Empirical [8] \\
\hline$C_{L_{q}}$ & $/ \mathrm{rad}$ & 7.95 & 10.71 & \\
\hline
\end{tabular}

Table IV shows a close match for the majority of parameters, with the exception of $C_{n_{\beta}}$, which was calculated to be more than double the published value. As was discussed earlier, this had the effect of changing the spiral mode of the aircraft significantly since the yaw contribution of the tail due to sideslip is a dominant factor in the spiral mode. Overall the calculated parameters were within an acceptable range for preliminary analysis, and neither empirical equations nor the computerized $3 \mathrm{D}$ vortex lattice code used showed any significant signs of being more accurate than the other. Rather, each method is useful in calculating parameters which the other is less proficient, for instance the empirical equations are particularly poor at calculating the lift-curve slope for wings of aspect ratio greater than 8. However, the vortex lattice codes excel in this area. Conversely, the empirical methods allow for the calculation of a much greater range of parameters than the vortex lattice method is capable of.
Looking into the future, flight testing of the UA is required in order verify the aircraft dynamics model and determine its accuracy. To ensure the highest accuracy without incurring large cost, the Differential Global Positioning System (DGPS) techniques detailed extensively in [10] will be used. By post-processing the raw pseudorange data from the satellites, a position accuracy of $2-5 \mathrm{~m}$ (or better using carrier-phase) can be consistently achieved, which will allow a trajectory of sufficient accuracy to be determined. For this, at least two GPS units capable of logging raw pseudo-range and timing data are required one at a stationary point of known position on the ground (the reference receiver - often at a survey point), and another on the UA itself. As long as the UA remains relatively close to the reference receiver, it is possible to take advantage of the spatial correlation of the GPS errors and remove them from the measurement [10]. In order to address navigation integrity requirements that apply to a variety of UA operational tasks, Avionics-Based Integrity Augmentation (ABIA) system will be adopted [11 - 14]. 


\section{CONCLUSIONS AND FUTURE WORK}

The lack of reliable 6-DoF aircraft dynamics models, especially for small UA, can be addressed through aftermarket development of dynamics models by using reverse engineering techniques. These techniques, combined with implementation of 6-DoF dynamics models as virtual navigation and guidance sensors, potentially provide a lowcost, low-weight/volume option for improving the performance, safety and reliability of small UA [15 - 19]. The qualitative validation of the method for determination of aerodynamic parameters was performed on the JAVELIN UA and on the AEROSONDE UA. The 6-DoF models developed for each UA were used to simulate trajectories which were compared against the published 6-DoF data available in the literature. In both cases, the trajectories were found to be rather similar, though an inaccurate estimation of $C_{n_{\beta}}$ had adverse effects on the spiral mode of the UA. The aerodynamic coefficients presented in this paper will need to be verified and their accuracy improved for consistent and reliable use as a virtual sensor. Wind tunnel testing is required to achieve this as well as generate reliable engine and propeller data for the same purpose. Flight testing, when conducted, will provide a basis for comparison as well as a method for further improvement of the aerodynamic derivatives through analysis of the aircraft response outside of laboratory conditions. Flight test of the JAVELIN UA will be conducted to verify the performance of the 6-DoF aircraft dynamics model acting as a virtual sensor onboard a small UA. The JAVELIN UA 6-DoF model will be adopted for developing the Next-Generation Flight Management System (NG-FMS) and for Air Traffic Management (ATM) studies [20, 21]. Additionally, ongoing research activities on obstacle avoidance will consider the JAVELIN UA as part of evaluating host and intruder platform dynamics [22-25].

\section{REFERENCES}

[1] J. McCormick, "Development of UAS in civil airspace and challenges for CASA," 2013. [Online]. Available: Error! Hyperlink reference not valid.

[2] V. Raja and K.J. Fernandes, Reverse Engineering: An Industrial Perspective,Springer-Verlag London Ltd, London, UK, 2008.

[3] R. Sabatini, S. Ramasamy, A. Gardi, and L.R. Salazar, "Low-cost Sensors Data Fusion for Small Size Unmanned Aerial Vehicles Navigation and Guidance," International Journal of Unmanned Systems Engineering, vol. 1, no. 3, pp. 16-47, 2013.

[4] R. Sabatini, M. Richardson, C. Bartel, T. Shaid, and S. Ramasamy, "A Low-cost Vision Based Navigation System for Small Size Unmanned Aerial Vehicle Applications," Journal of Aeronautical Engineering, vol. 2, no. 2, 2013.

[5] Unmanned Dynamics LLC, AeroSim User's Guide Version 1.2, Hood River, OR, USA.

[6] Wikipedia, "AAI Corporation AEROSONDE", Wikimedia Foundation, 2013. [Online]. Available: Error! Hyperlink reference not valid. [Accessed $29^{\text {th }}$ September, 2013].

[7] CREAFORM, "Technical Specifications: Handyscan Handheld 3D Scanner," CREAFORM, 2013. [Online].

Available: $\quad$ http://www.creaform3d.com/en/metrology-solutions /products/portable-3d-scanner/technical-specifications-handyscan-3d [Accessed 29 ${ }^{\text {th }}$ September, 2013].

[8] M. Napolitano, Aircraft dynamics: from modeling to simulation, John Wiley \& Sons, Inc., Hoboken, NJ, USA, 2012.
[9] A. Deperrois, "XFLR5". [Online]. Available: Error! Hyperlink reference not valid. [Accessed $29^{\text {th }}$ September, 2013]

[10] R. Sabatini and G.B. Palmerini, RTO AGARDograph AG-160 Vol. 21 : Differential Global Positioning System (DGPS) for Flight Testing, NATO Science and Technology Organisation, 2008.

[11] R. Sabatini, T. Moore, and C. Hill, "A New Avionics-Based GNSS Integrity Augmentation System: Part 1 - Fundamentals", Journal of Navigation, vol. 66, no. 3, pp. 363-384, 2013.

[12] R. Sabatini, T. Moore, and C. Hill, "A New Avionics-Based GNSS Integrity Augmentation System: Part 2 - Integrity Flags", Journal of Navigation, vol. 66, no. 4, pp. 501-522, 2013.

[13] R. Sabatini, T. Moore, and C. Hill, "A Novel GNSS Integrity Augmentation System for Civil and Military Aircraft", International Journal of Mechanical, Industrial Science and Engineering, vol. 7, no. 12, pp. 1433-1449, WASET, 2013.

[14] R. Sabatini, T. Moore, and C. Hill, "Avionics Based GNSS Integrity Augmentation for Mission- and Safety-Critical Applications", in proceedings of $25^{\text {th }}$ International Technical Meeting of the Satellite Division of the Institute of Navigation: ION GNSS-2012. Nashville, TN, USA, 2012.

[15] R. Sabatini, C. Bartel, A. Kaharkar, and T. Shaid, "Low-cost Vision Sensors and Integrated Systems for Unmanned Aerial Vehicle Navigation and Guidance", ARPN Journal of Systems and Software, ISSN: 2222-9833, vol. 2, no. 11, pp. 323-349, December 2012.

[16] R. Sabatini, L.R. Salazar, A. Kaharkar, C. Bartel, and T. Shaid, "Carrier-phase GNSS Attitude Determination and Control System for Unmanned Aerial Vehicle Applications", ARPN Journal of Systems and Software, ISSN: 2222-9833, vol. 2, no. 11, pp. 297-322, November 2012.

[17] R. Sabatini, C. Bartel, A. Kaharkar, T. Shaid, and S. Ramasamy, "A Novel Low-cost Navigation and Guidance System for Small Unmanned Aircraft Applications", paper presented at the WASET International Conference on Aeronautical and Astronautical Engineering, Melbourne, Australia, December 2013.

[18] R. Sabatini, A. Kaharkar, C. Bartel and T. Shaid, "Carrier-phase GNSS Attitude Determination and Control for Small UAV Applications", Journal of Aeronautics and Aerospace Engineering, vol. 2, no. 4, July 2013.

[19] R. Sabatini, M.A. Richardson, C. Bartel, A. Kaharkar, T. Shaid, L.R Salazar, and A. Gardi, "A Low-cost Vision Based Navigation System for Small Size Unmanned Aerial Vehicle Applications", Journal of Aeronautics and Aerospace Engineering, vol. 2, no. 3, May 2013.

[20] A. Gardi, R. Sabatini, S. Ramasamy, and K. de Ridder, "4Dimensional Trajectory Negotiation and Validation System for the Next Generation Air Traffic Management", in proceedings of AIAA Guidance, Navigation \& Control Conference 2013 (GNC 2013), Boston, MA, USA, 2013.

[21] S. Ramasamy, R. Sabatini, A. Gardi, and Y. Liu, "Novel Flight Management System for Real-Time 4-Dimensional Trajectory Based Operations", in proceedings of AIAA Guidance, Navigation \& Control Conference 2013 (GNC 2013), Boston, MA, USA, 2013.

[22] R. Sabatini, A. Gardi, and M.A. Richardson, "LIDAR Obstacle Warning and Avoidance System for Unmanned Aircraft", International Journal of Mechanical, Industrial Science and Engineering, vol. 8, no. 4, pp. 62-73, WASET, 2014.

[23] L.R. Salazar, R. Sabatini, S. Ramasamy, and A. Gardi, "A Novel System for Non-Cooperative UAV Sense-And-Avoid", in proceedings of European Navigation Conference 2013, Vienna, Austria, 2013.

[24] R. Sabatini, A. Gardi, and S. Ramasamy, "A Laser Obstacle Warning and Avoidance System for Manned and Unmanned Aircraft," in proceedings of IEEE 1st International Workshop on Metrology for Aerospace 2014, Benevento, Italy, 2014.

[25] S. Ramasamy, R. Sabatini, and A. Gardi, "Avionics Sensor Fusion for Small Size Unmanned Aircraft Sense-and-Avoid", in proceedings of IEEE 1st International Workshop on Metrology for Aerospace 2014, Benevento, Italy, 2014. 